\title{
Surveillance Bias
}

National Cancer Institute

\section{Source}

National Cancer Institute. Surveillance Bias. NCI Thesaurus. Code C50890.

A phenomenon involving the cancer diagnosis of a family member or friend that heightens awareness of the disease. Subsequently, this awareness results in an increased detection of asymptomatic tumors because medical attention is sought. This cycle is termed surveillance bias. 